\title{
Planetary nebula spectra in M60 and M 82
}

\author{
Roberto H. Méndez ${ }^{1}$, Ana M. Teodorescu ${ }^{1}$, Takashi Hattori ${ }^{2}$, \\ Laura Magrini ${ }^{3}$ and Lent C. Johnson ${ }^{4}$ \\ ${ }^{1}$ Institute for Astronomy, University of Hawaii, \\ 2680 Woodlawn Drive, Honolulu, Hawaii 96822, USA \\ email: mendez@ifa.hawaii.edu, ana@ifa.hawaii.edu \\ ${ }^{2}$ Subaru Telescope, Nat. Astr. Obs. of Japan, Hilo, Hawaii, USA \\ ${ }^{3}$ INAF-Osservatorio Astrofisico di Arcetri, Firenze, Italy \\ ${ }^{4}$ Dept. of Astronomy, University of Washington, Seattle, USA
}

\begin{abstract}
Using the Subaru telescope and its FOCAS spectrograph in multi-object mode, we have obtained spectra of a selection of bright PNs in two galaxies: the Virgo giant elliptical M 60 (NGC 4649), and the starburst spiral M 82. We report on individual extinctions, as determined from the Balmer decrement, and also on the intensities of [O III] 4959 and 5007 relative to $\mathrm{H} \beta$.
\end{abstract}

Keywords. galaxies: individual (NGC 4649, M 82), dust, extinction, planetary nebulae: general

\section{Introduction}

Abundance determinations in extragalactic PNs are difficult and time consuming because some key diagnostic emission lines, like [O III] 4363, are very faint, particularly at high metallicity. Previous work (Méndez et al. 2005) in the elliptical galaxy NGC 4697 showed several PNs with very strong [O III] 5007, about 20 times stronger than $\mathrm{H} \beta$. Such high relative intensities require at least solar metallicity. This means that, in principle, a useful test for high metallicity could consist of detecting $\mathrm{H} \beta$, which is much easier than detecting [O III] 4363. We have tried to explore this idea by observing selected bright PNs in M 60, previously discovered by Teodorescu et al. (2011). For comparison, we include a selection of bright PNs in M 82, previously discovered by Johnson et al. (2009).

\section{Observations}

FOCAS was used with the dispersing element 300B, which gives a resolution of about 500 for slits 1 arcsec wide. We observed 2 masks in M 82, obtaining 9 and 11 1800-s exposures through masks 1 and 2, respectively, in January 2010. We obtained 28 2000-s exposures of M 60 through a single mask in May 2010. The spectrophotometric standard was, in all cases, HZ44 (Oke 1990). Because of space limitations we have omitted the tables of observed relative intensities. A paper with a full description is in preparation. We obtained spectra of 13 and 19 PNs in M 60 and M 82, respectively.

\section{Foreground and internal extinctions in $\mathrm{M} 82$}

The foreground extinction value for M 82 is 0.28 at 5007 (Johnson et al. 2009). Expressing this in terms of the logarithmic extinction $c$ at $\mathrm{H} \beta$, we find that the foreground $c=0.4 A \beta=A_{5007} / 2.54=0.11$. We can then compare with the individual values of $c$ from the Balmer decrement in our $19 \mathrm{PN}$ spectra. Assuming an unreddened value of 2.85 
for the ratio $\mathrm{H} \alpha / \mathrm{H} \beta$, we find that the average $c$ from our 19 PNs is 0.23 . Only a few PNs in our M 82 sample show a significant reddening.

\section{Foreground and internal extinctions in $M 60$}

The foreground extinction for M 60 is $c=0.04$ (from NED, see Schlegel et al. 1998). Our $13 \mathrm{PNs}$ give an average $c=0.42$. This is unexpectedly high. If we were to uniformly correct the observed PN fluxes for the effect of this internal extinction, then the distance estimated using the PN luminosity function (PNLF distance) would become shorter, increasing the already large unexplained discrepancy with the SBF distance, as discussed by Teodorescu et al. (2011). This certainly creates a motivation to enlarge the sample and acquire better individual PN spectra in M 60 and other elliptical galaxies.

\section{The intensities of [O III $] 4959$ and 5007}

The PNs in M 60 show a high frequency (6 out of 13 PNs) of very strong [O III] 5007, of the order of 20 times $\mathrm{H} \beta$. A similar behavior was found earlier in NGC 4697 (Méndez et al. 2005). The contrast with M 82 is interesting: only two out of 19 PNs in M 82 show such extreme 5007 intensities. In M 60, some of the strong 5007 objects are located far from the center of the galaxy; the same thing happened in NGC 4697.

This behavior can have an impact on how we model the PNLF. If old, metal-rich populations show a tendency to populate the bright end of the PNLF with very strong $5007 \mathrm{PNs}$, this might help to somehow relax the requirement of relatively high central star masses, which has always been a problem (see for example Méndez et al. 2008). Again this emphasizes how important it will be to obtain better individual PN spectra in many distant galaxies to better understand the PNLF.

\section{Acknowledgements}

This work has been supported by the NSF under grant 0807522 .

\section{References}

Johnson, L. C., Méndez, R. H., \& Teodorescu, A. M. 2009, ApJ, 697, 1138

Méndez, R. H., et al. 2005, ApJ, 627, 767

Méndez, R. H., et al. 2008, ApJ, 681, 325

Oke, J. B. 1990, AJ, 99, 1621

Schlegel, D. J., Finkbeiner, D. P., \& Davis, M. 1998, ApJ, 500, 525

Teodorescu, A. M., et al. 2011, ApJ, 736, 65 